\title{
Moments of Ambient Doppler Spectra
}

\author{
S. K. Lehman
}

March 23, 1993

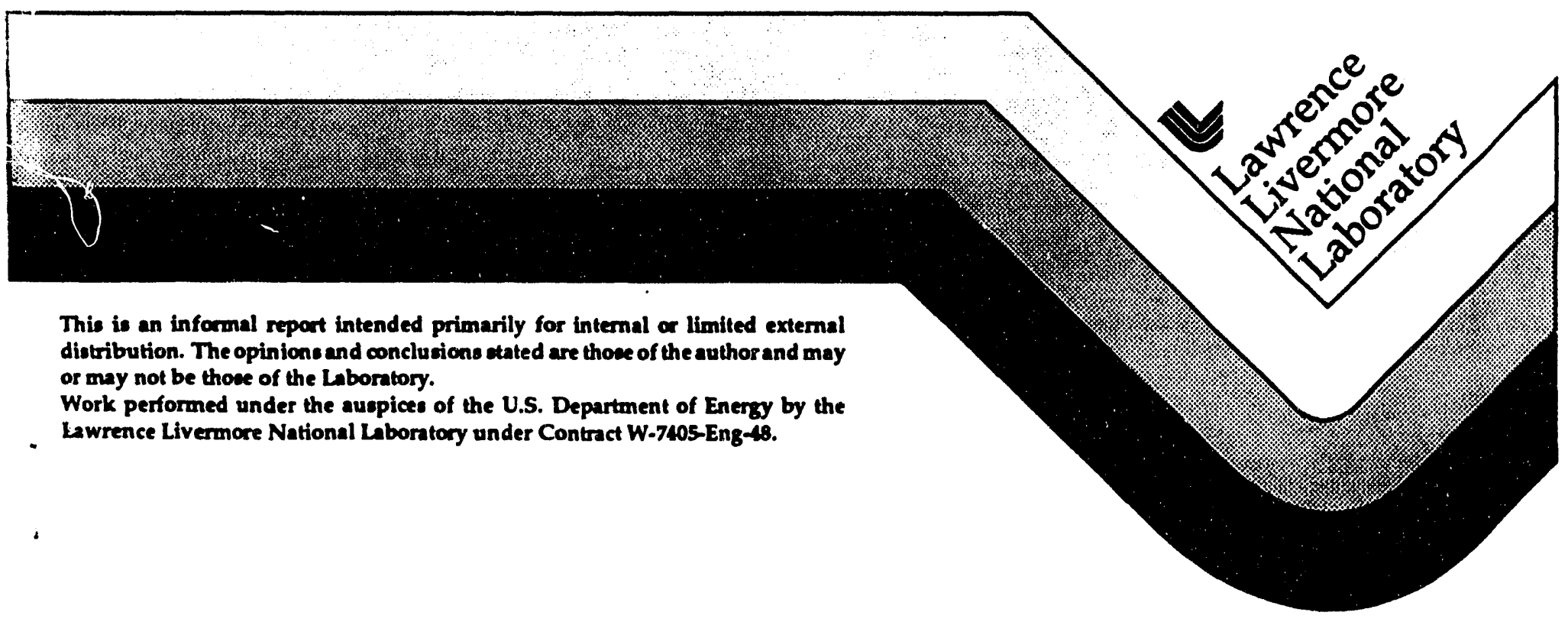

\section{MASTER}


This document was prepared as an account of work sponsored by an agency of the Uniled States Covernment. Neither the Uniled States Government nor the University of California nor any of their employees, makes any warranty, express or implied, or assumes any legal liability or responsibility for the accuracy, completeness, or usefulness of any information, apparatus, product, or process disclosed, or represents that its use would not infringe privately owned rights. Reference herein to any specific commercial products, process, or service by trade name, trademark, manufacturer, or otherwise, does not necessarily constitute or imply its endorsement, recommendation, or favoring by the United States Government or the University of California. The views and opinions of authors expressed herein do not necessarily state or reflect those of the United Stales Government or the University of California, and shall not be used for advertising or product endorsement purposes.

This report has been reproduced
directly from the best avallable copy.

Available 10 DOE and DOE contractors from the Olfice of Scientific and Technical Information P.C. Bon 62, Oak Ridge, TN 3783!

Prices available from 1615) 576-6401, FT5 626-8401.

Available to the public from the

National Technical Information Service

U.S. Department of Commerce

5285 Port Royal Rd.

Springfield, VA 22161

Price

Code

A01

Microfiche

Papercopy Prices

$\mathrm{AO2}$

A03

A04

A05

A06

A07

A.08

A09

A10

A11

A12

A13

A14

A 15

A16

A 17

A18

A 19

A 20

A21

A 22

A23

A24

A25

A99
1- 10

$11-50$

51- 75

76-100

101-125

126-150

151-175

176-200

201-225

226-250

251-275

276-300

301-325

326-350

351-375

376-400

401-425

426-450

451-475

476-500

501-525

526-550

551-575

576-600

601 \& UP 


\title{
Moments of Ambient Doppler Spectra*
}

\author{
S. K. Lehman \\ Iniversity of California \\ Lawrence Livermore National Laboratory \\ Livermore. C'A 94.5 .50
}

March 22, 1993

\begin{abstract}
We studied the first four moments (center of mass, standard deviation. skew, and kurtosis) of the Doppler spectra in ambient regions of LLNL-Hughes real aperture radar data collected during WCSEX91-92. Our goal was to correlate trends in the moments with wind velocity and direction.

Although the center of mass appears to increase when the wind is blowing into the radar antenna, no other conclusions have been drawn from the higher order moments.
\end{abstract}

\section{Contents}

1 Definition of Continuous and Discrete Moments 1

2 Doppler Spectrum $\quad 2$

3 Results and Conclusions $\quad 3$

A Justification of Doppler Unwrapping 5

A.1 Manufactured Doppler Spectrum . . . . . . . . . . . . . . . . . . . . . . . . . . . . )

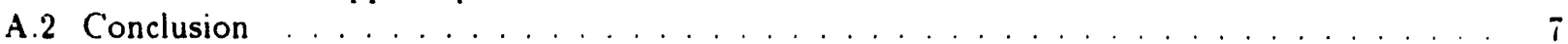

\section{Definition of Continuous and Discrete Moments}

Let $g(x)$ be a continuous function defined over the support $[a, b]$ with $g(x) \geq 0$ for $x \in[a, b]$. The $k^{\text {th }}$ moment of $f(x)$ is defined as:

$$
u_{k}=\int_{a}^{b} x^{k} g(x) d x
$$

The zeroth moment is the mass:

$$
u_{0}=\int_{a}^{b} g(x) d x
$$

The first moment is the center of mass. $\mu$ :

$$
\mu=\frac{1}{\mathcal{M}_{0}} \int_{a}^{t} x g(x) d x
$$

- Work performed under the auspices of Z.S. Department of Energy by the Lawrence Livermore Nationa Laboratory under cuntract No. W-7405-E.VG-48 
The central moments are defined as the moments with respect to the center of mass:

$$
c_{k}=\frac{1}{. H_{11}} \int_{a}^{b}(x-\mu)^{k} g(x) d x
$$

where

$$
\begin{gathered}
c_{11}=\frac{1}{\cdot M_{0}} \int_{a}^{b} y(x) d x=1 \\
\mathcal{C}_{1}=\frac{1}{{ }^{M_{0}}} \int_{a}^{b}(x-\mu) g(x) d x=0
\end{gathered}
$$

In the work presented here, all moments above the first (center of mass) are central moments.

For the discrete case, let $D(n)$ be a discrete function defined over $n \in[0, \ldots-1]$ with $D(n) \geq 0$. 1..1 $D(n)$ have sample interval $\Delta x$ and origin $x_{0}$. The moments of $D(n)$ are defined as:

$$
\begin{aligned}
\mathcal{M}_{k} & =\frac{\Delta x}{M_{0}} \sum_{n=0}^{N-1}\left(x_{0}+n \Delta x\right)^{k} D(n) \\
\mathcal{C}_{k} & =\frac{\Delta x}{M_{0}} \sum_{n=0}^{N-1}\left(x_{0}+n \Delta x-\mu\right)^{k} D(n) \\
\mathcal{M}_{0} & =\Delta x \sum_{n=0}^{N-1} D(n) \\
\mu & =\frac{\Delta x}{M_{0}} \sum_{n=0}^{N-1}\left(x_{0}+n \Delta x\right) D(n)
\end{aligned}
$$

\section{Doppler Spectrum}

The Doppler spectrum is estimated over a few second's worth of data, and is the mean intensity of the Fourier transform of consecutive slices of size $V_{\text {dft }}$ through the data for a fixed range. Let $r(m . n)=$ $I(m, n)+i Q(m, n)$ be the complex IQ dat a where $0 \leq m \leq M-1$ is the range index. and $0 \leq n<x$ is the time index. Then the definition of the Doppler spectrum is:

$$
D(m, l)=\frac{1}{K} \sum_{k=0}^{K-1}\left|\mathcal{F}_{\ell}\left\{r_{k}(m, n) w(n)\right\}\right|^{2}
$$

where $K$ is the number of time slices through the data, $u(n)$ is a smoothing window. $l$ is the discrete frequency index, and $r_{k}(m, n)$ is the $k^{\text {th }}$ slice through the data, defined as:

$$
r_{k}(m, n)=r\left(m, n+k \cdot N_{\mathrm{dft}}\right) \quad 0 \leq n \leq N_{\mathrm{dft}}-1
$$

and $\mathcal{F}_{t}\{\}$ is the DFT of $r_{k}$ with respect to time:

$$
\mathcal{F}_{t}\left\{r_{k}(m, n)\right\}=\frac{1}{V_{\mathrm{dft}}} \sum_{n=0}^{N_{\mathrm{d} I t}-1} r_{k}(m, n)(-1)^{n} \exp \left(-2 \pi i \frac{n l}{V_{\mathrm{dft}}}\right)
$$

Note the multiplication by -1 in the DFT. This technique is known as "sign modulation" and has the effect of moving the origin in the frequency domain to the center of the data set. See [1] for a description of this procedure which we call "toggling".

In the discrete frequency domain. the sample interval is:

$$
\Delta f=\frac{1}{._{d f t} \Delta t}
$$


and. as a result of the toggling, the origin is:

$$
f_{0}=\frac{-1}{2 \Delta t}
$$

With the Doppler spectrum defined in (10), the moments were calculated from a mean spertrum in an ambient region. Thus. picking an $m^{\prime}$ in an ambient region, we computed a mall spectrum:

$$
\dot{D}(l)=\frac{1}{W} \sum_{m=m^{\prime}-W / 2}^{m^{\prime}+W^{\prime} / 2-1} D(m . l)
$$

and estimated the monents from $\dot{D}(l)$ :

$$
\begin{aligned}
& \cdot M_{k}=\frac{\Delta f}{. M_{0}} \sum_{l=0}^{N_{t / 1+1}-1}\left(f_{0}+l \Delta f\right)^{k} \dot{D}(l) \\
& \mathcal{C}_{k}=\frac{\Delta f}{M_{0}} \sum_{l=0}^{N_{1 / 1}-1}\left(f_{0}+(\Delta f-\mu)^{k} \dot{D}(l)\right. \\
& \mathcal{M}_{0}=\Delta f \sum_{l=0}^{N_{\text {dit }}-1} \dot{D}(l) \\
& \mu=\frac{\Delta f}{. M_{0}} \sum_{l=0}^{N_{i n-1}-1}\left(f_{0}+l \Delta f\right) \dot{D}(l)
\end{aligned}
$$

Note if $\dot{D}(l)$ went negative, that is $\min _{l}[\dot{D}(l)]<0$, we shifted it positive by subtracting $\min _{l}[\dot{D}(l)]$ :

$$
\dot{D}(l) \longrightarrow \tilde{D}(l)-\min _{l}[\dot{D}(l)]
$$

For the data presented here, the Doppler spectra were estimated over 30 seconds of data with a time slick. size of $N_{\mathrm{dft}}=128$ returns. The number of range points over which the meall spectra were computed wer. $H=5$.

Unfortunately, due to the relatively long temporal sample interval in the LL.VL-Hughes RAR. some of the Doppler spectra are aliased. To eliminate this problem. we judiciously "unwrapped" the spectra. taking into account the wind direction with respect to the radar and using the fact the spectra are band limited. That is, we estimated where the upper limit of the aliased spectrum ended, then cut and barrel shifted the data from that frequency. Figure( 1) shows data from the 1991 experiment overlayed with its unwrapped spectrum. The cut was made around $-53 \mathrm{~Hz}$ as indicated by the arrow. Appendix A provides a .Mathematera if not mathematical justification for this procedure.

\section{Results and Conclusions}

The graphs in Figures (2) through (5). show the first four moments (center of mass. $\mu$ : standart deviation. $\sigma=\sqrt{\mathcal{C}_{2}}$; cubed root of skew, $\sqrt[3]{\mathcal{C}_{3}}$; and the fourth root of kurtosis, $\sqrt[4]{\mathcal{C}_{4}}$ ) for $\mathrm{HH}$ and $\mathrm{VV}$ as a function of wind direction with respect to the radar and wind velocity. Wind direction is across the horizontal axis and velocity is on the vertical. A direction of $0^{\circ}$ is into the radar while $180^{\circ}$ is from the back. The third axis is the specified moment represented by the colored dots. The lighter the color. the higher the valur of thr. moment. The mapping of the color scale is given at the bottom of each plot.

For comparison. We show two cases: 1) we have included unwrapped spectra (Figures (2) and (3)). and. 2) we have no unwrapped (thus no aliased) spectra (Figures (4) and (5)). The plots tend to agree thus supporting the unwrapping. 


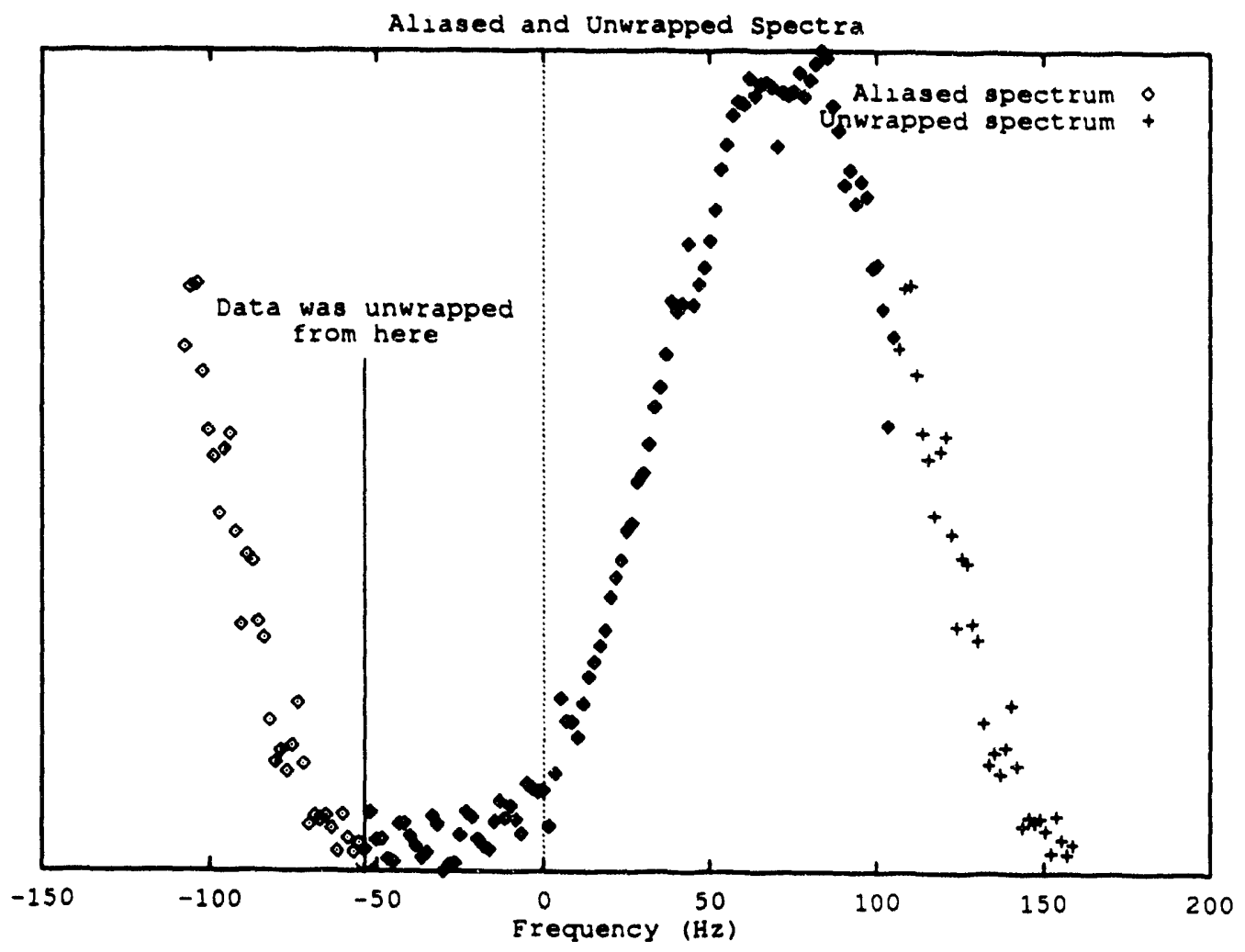

Figure 1: Example of aliased and unwrapped spectra 


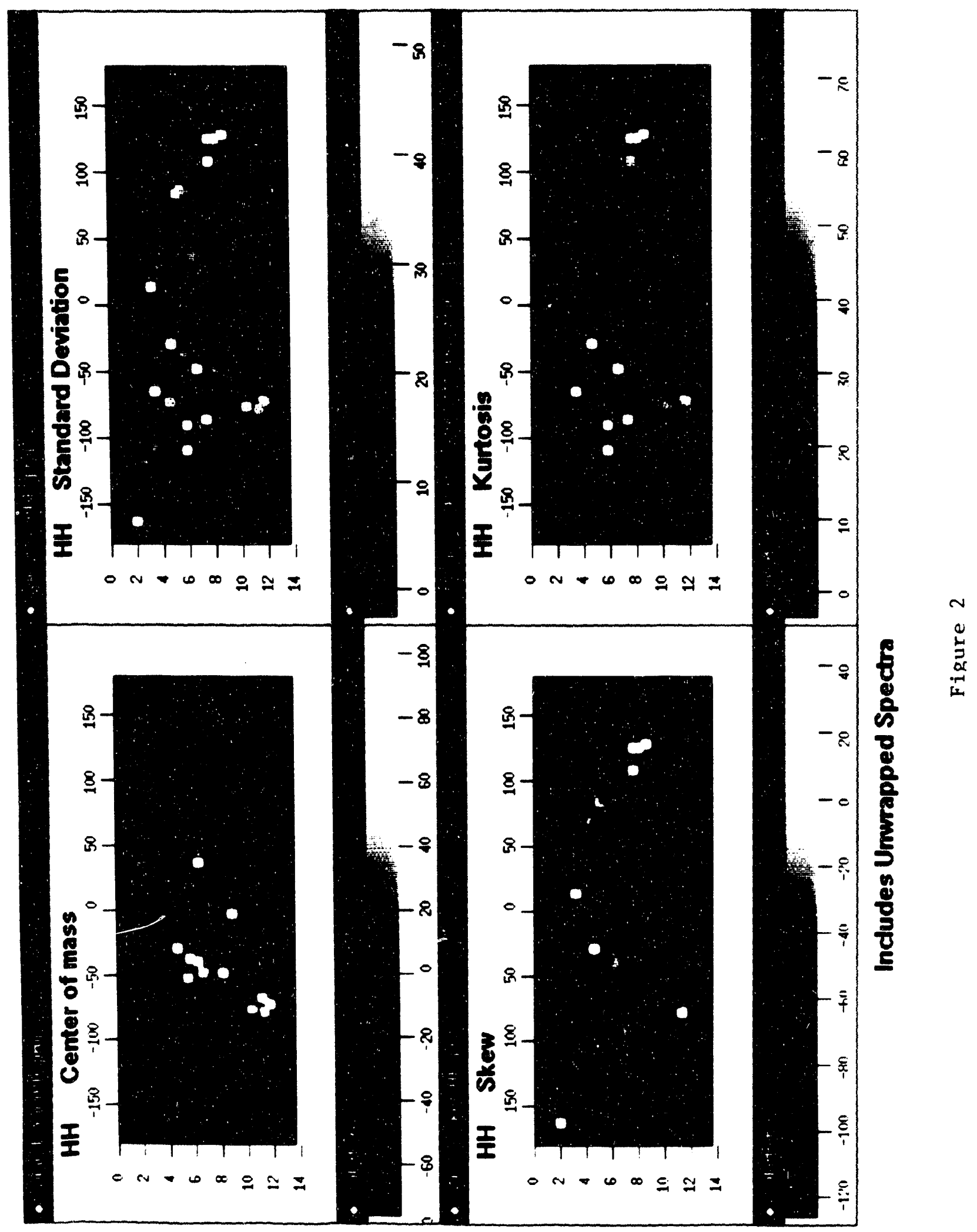




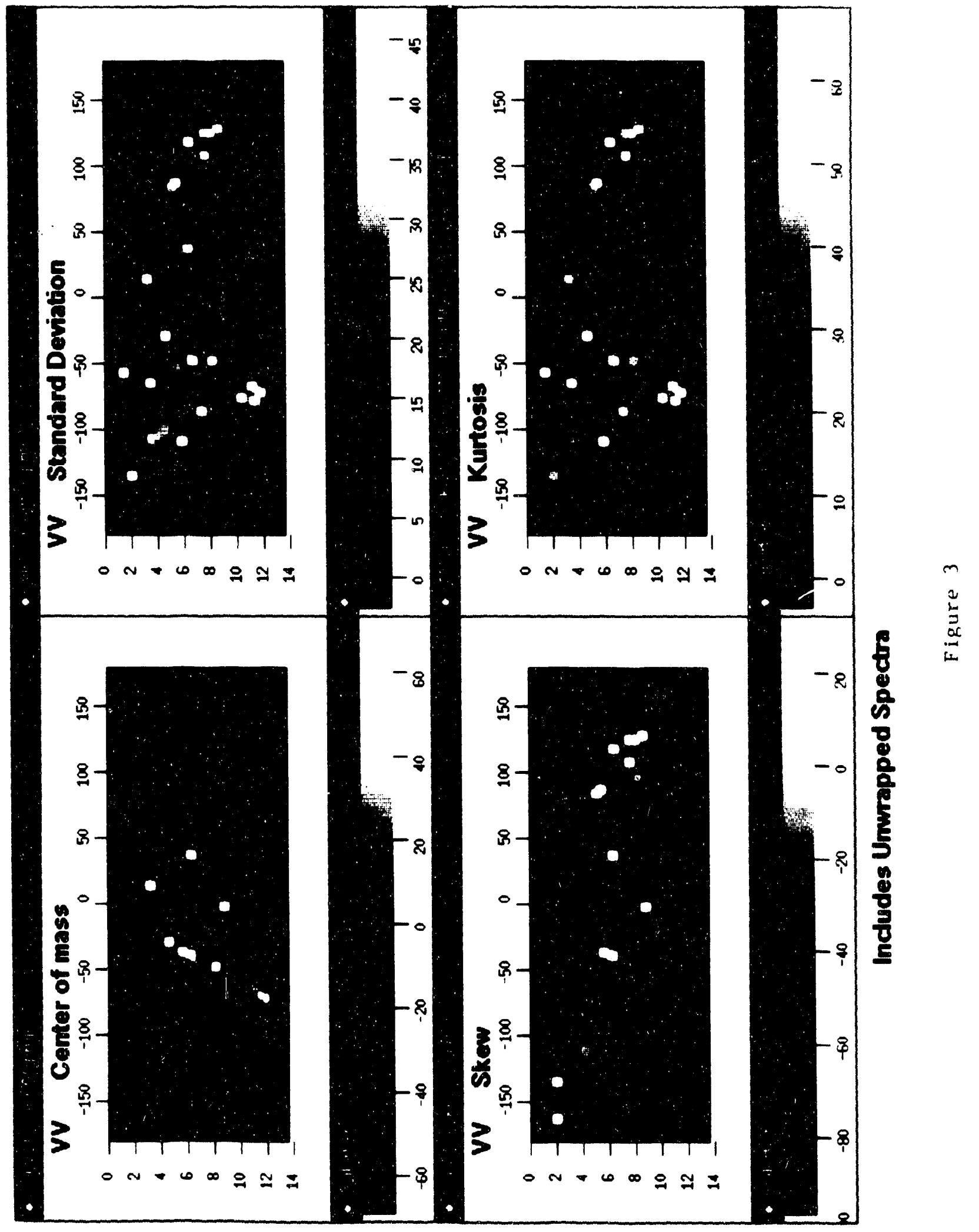




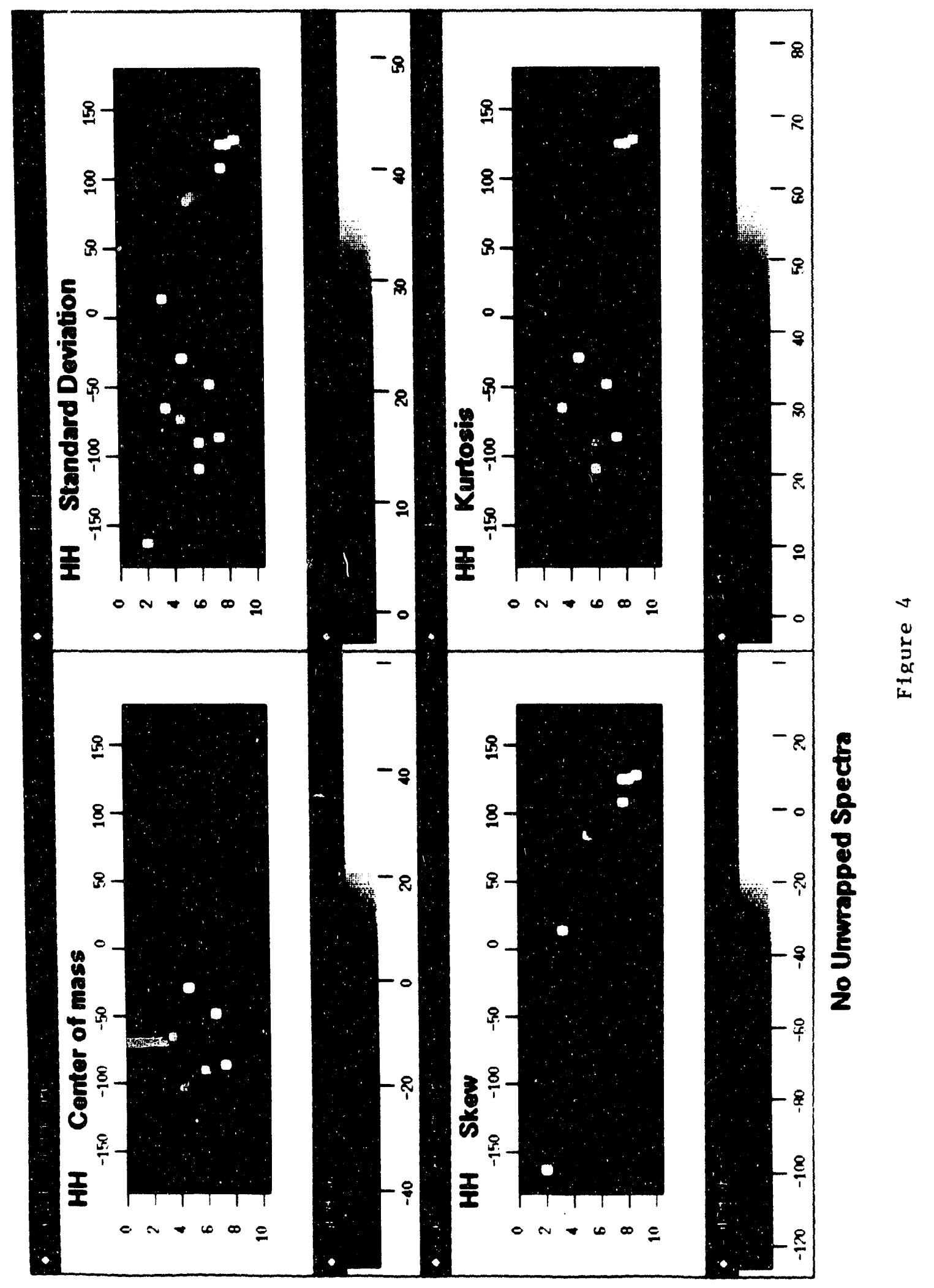




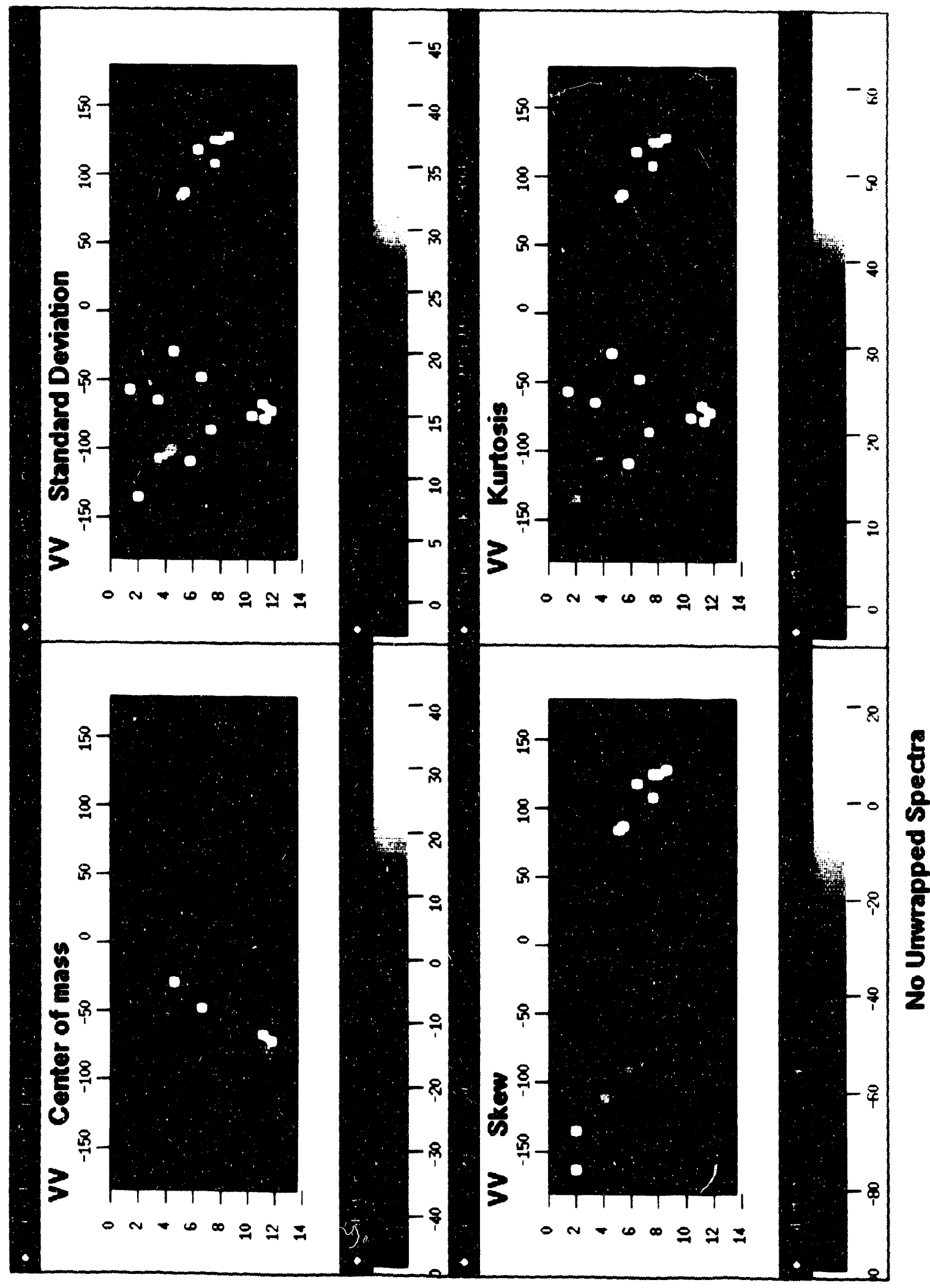


The easiest to interpret is the center of mass. We expect to have the highest center of mass (in frequenc $y$ ) when the wind is blowing into the radar and the lowest or most negative when the wind is from behind. 'This causes wind driven currents toward and away from the radar respectively, and is confirmed in the graphs.

We are currently developing models for the Doppler spectra and further interpretation of the data will have to be performed when these have beell implemented.

\section{A Justification of Doppler Unwrapping}

In working with the Doppler spectra of the LL.NL-Hughes RAR. we were obliged to "unwrap" spectra aliased because of the under sampling of the radar. Under the normal Nyquist sampling constraint, aliased data cannot be recovered. However, using the additional knowledge of wind direction, the band-limited nature of the spectra. and the location of the centroid is within a band of $\pm 150 \mathrm{~Hz}$. we chose an appropriate place to cut and unwrapped the spectra. A question arose to the validity of this procedure.

We present here a Mathematica if not mathematical justification why the aliased Doppler spectra from IVCSEX91 and WCSEX92 may be unwrapped, in fact "unaliased", using prior knowledge.

\section{A.1 Manufactured Doppler Spectrum}

We manufactured the following Doppler spectrum:

$$
D(f)=\frac{1}{\sigma \sqrt{2 \pi}} \exp \left[\frac{1}{2}\left(\frac{f-f_{c}}{\sigma}\right)^{2}\right]
$$

where $f_{c}=100$ and $\sigma=25$ are the Doppler centroid and standard deviation respectively and are based upon the actual values taken from the 1991-92 data for the case of the wind blowing into the radar.

The analytical Fourier transform of $D(f)$ is:

$$
S(t)=\exp \left[-2 \pi i f_{\mathrm{s}} t\right] \exp \left[-2(\sigma \pi t)^{2}\right]
$$

The Fourier transform pair. $D(f)$ and $S(t)$, are plotted in Figure 6 .

In 1991, the time sample interval. $\Delta t$, was approximately 6 milliseconds or $16 \mathrm{t} \mathrm{Hz}$. In 19y2, it was 3.j) milliseconds or $286 \mathrm{~Hz}$. Notice a choice for the cutoff frequency of the manufactured Doppler spectrum is above $200 \mathrm{~Hz}$. Thus, to sample the time domain signal correctly, we would require a sample frequency above $400 \mathrm{~Hz}$ based upon the Nyquist criteria.

In Doppler processing the LLNL-Hughes RAR data. time slices of $N=128$ points were used. Thus. sampling with a time interval oi $\Delta t$ results in a sample rate of $f_{s}=1 / \Delta t$ and a sample interval in the frequency domain of $\Delta f=1 /(N \Delta t)$. Sampling the analytical time domain signal $S(t)$ symmetrically about the origin results in a start time of $t_{0}=-N \Delta t / 2$. Before performing the DFr. the time samples are sign modulated (or "toggled") which moves the frequency domain origin to the center of the series. Thus the starting frequency of the sampled Doppler spectrum is $-1 /(2 \Delta t)$.

We present here three cases:

- $S(t)$ is sampled at the 1991 rate of $6 \mathrm{~ms}$ :

- $S(t)$ is sampled at the 1992 rate of $3.5 \mathrm{~ms}$ :

- $S(t)$ is sampled at $2.5 \mathrm{~ms}=400 \mathrm{~Hz}$ (twice a cut off frequency of $200 \mathrm{~Hz}$ ). which we call a "Sanity ('herk" and is used to verify the correct functioning of the Mathematica code.

Table 1 lists the various parameters discussed above for each case.

Figure 7 shows the real and imaginary parts of $S(t)$ and its sampled version at the 1991 sample interval of 6 milliseconds. Note the undersampling. Figure 8 shows the DFT of the sampled signals in Figure 7 plotted with the manufactured Doppler spectrum. The aliasing is obvious. With the knowledge the true spectrum is band limited and the wind is blowing into the radar. we know the negative frequency components are aliased 


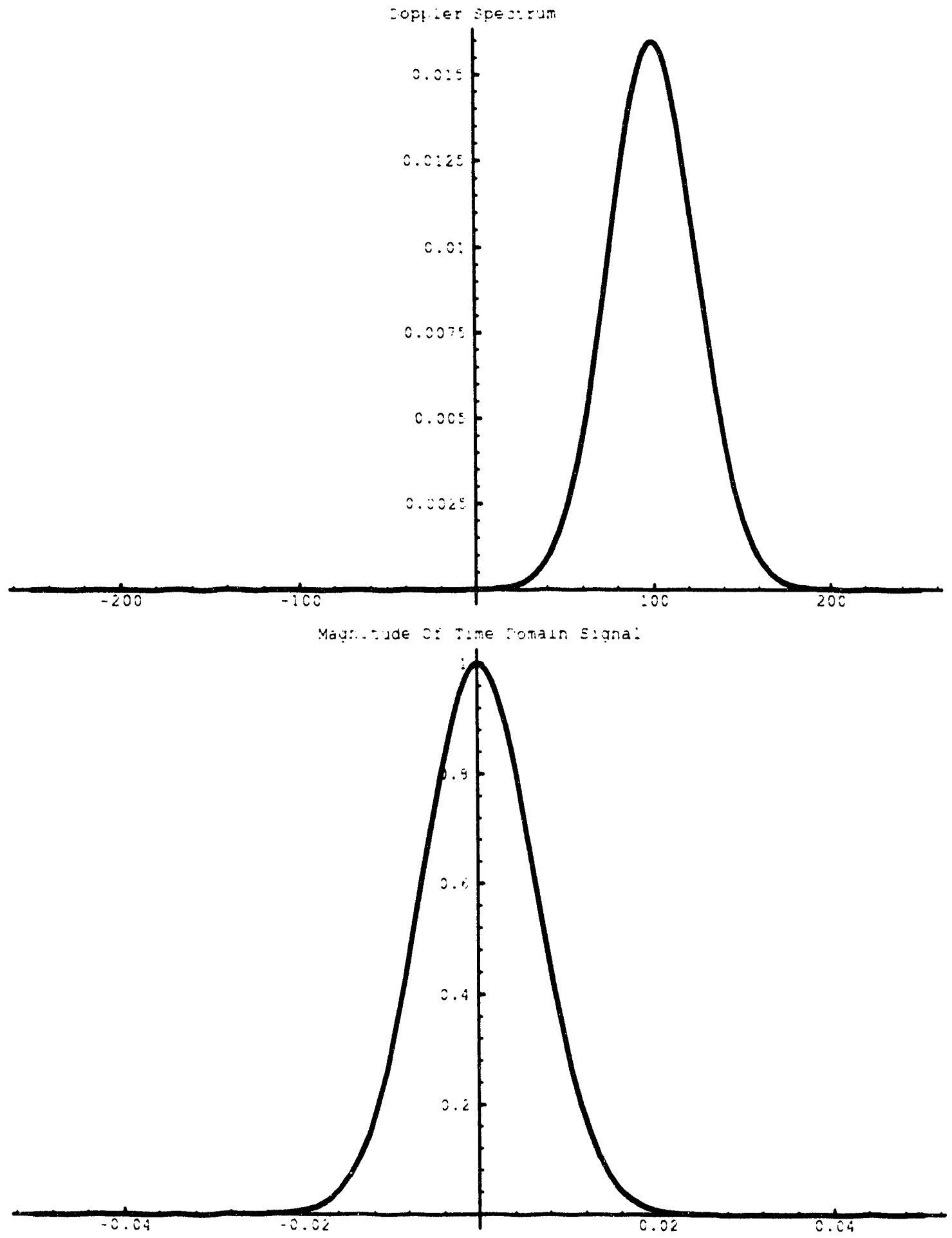

Figure 6: Manufactured Doppler spectrum (top) and the magnitude of its Fourier transform (bottom). The centroid and standard deviation of the manufactured Doppler spectrum were chosen to match actual data.

10 


\begin{tabular}{|c|l|l|l|}
\hline PARAMETER & 1991 & 1992 & Sanity Check \\
\hline$f_{s}(\mathrm{~Hz})$ & $16 i$ & 286 & 400 \\
$J_{t}(\mathrm{~ms})$ & 6 & 3.5 & 2.5 \\
$t_{0}(\mathrm{~s})$ & -0.384 & $-(0.224$ & -0.16 \\
$د f(\mathrm{~Hz})$ & 1.3 & 2.2 & 3.125 \\
$f_{0}(\mathrm{~Hz})$ & -8.3 & -143 & -200 \\
\hline
\end{tabular}

Table 1: Sampling frequency. $f_{s}$. sampling interval. $\Delta t$. sampling origin. $t_{0}$. frequency interval. $\Delta f$. and frequency origin. $f_{0}$. of the test cases. For all cases there are 128 sample points.

and should be moved into positive frequency bins. Thus, we chose to cut the sampled spectrum where it is close to zero, ai around $11 \mathrm{~Hz}$. and "unwrap" the spectrum. Figure 9 shows the true and unwrapped spectra. The match is very good. If we define an error as:

$$
e=\sqrt{\sum_{n=0}^{N-1}\left[D\left(f_{w}+n \Delta f\right)-l^{\prime}(n)\right]^{2}}
$$

where $l(n)$ is the unwrapped spectrum and $f_{w}$ is the frequency about which the spectrum unwrapped. then for 1991 , the error is $2.6 \times 10^{-4}$.

Figures 10,11. and 12. show the same procedure applied using the 1992 sample interval of 3.5 milliseconds. For this case the unwrapping cut was made at $0 \mathrm{~Hz}$, and the error is $7.4 \times 10^{-5}$.

Finally, the sanity check is in Figures 13 and 14. We are relieved to see there is no aliasing and the liscrete and sampled spectra match very well. Thus the Mathematica procedure functions as expected. The error is $6.7 \times 10^{-6}$. and is defined as:

$$
\epsilon=\sqrt{\sum_{n=0}^{N-1}\left[D\left(f_{u}+n \Delta f\right)-D_{d}(n)\right]^{2}}
$$

where $D_{d}(n)$ is the discrete. correctly sanipled spectrum.

\section{A.2 Conclusion}

We presented a simple analytical model for a Doppler spectrum with parameters based upon actual data. and its time domain counterpart. We sampled the spectrum at the rates used in the LLNL-Hughes RAR for ihe WCSEX91-92 experiments and demonstrated the ability to recover aliased data by "unwrapping" the discrete spectrum. Finally. we presented a "sanity check" on the procedure to verify its functioning.

\section{References}

[1] E. M. Johansson and J. P. Fitch. Remarks on Translation by Sign Morlulation. IEEE Trans. Signal Processing. April 1991 

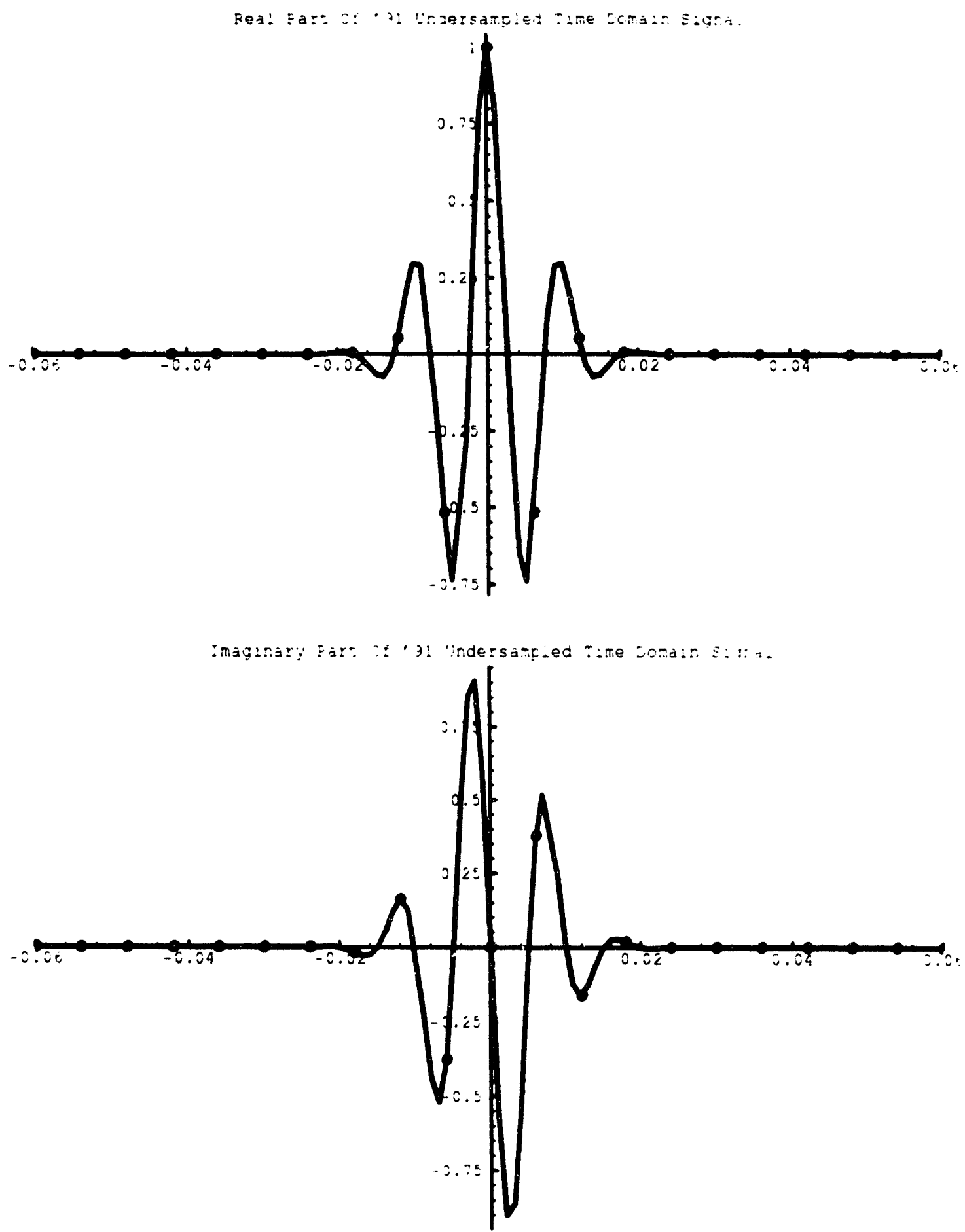

Figure 7 : Real and imaginary parts of the analytic time domain signal. $S(t)$, and the discrete signal sampuled at the 1991 interval of $6 \mathrm{~ms}$. Note: only the center part of the signal is shown. there are actually 12r puint. which have been truncated from the graph because they are zero. 


$$
\frac{u}{n}
$$



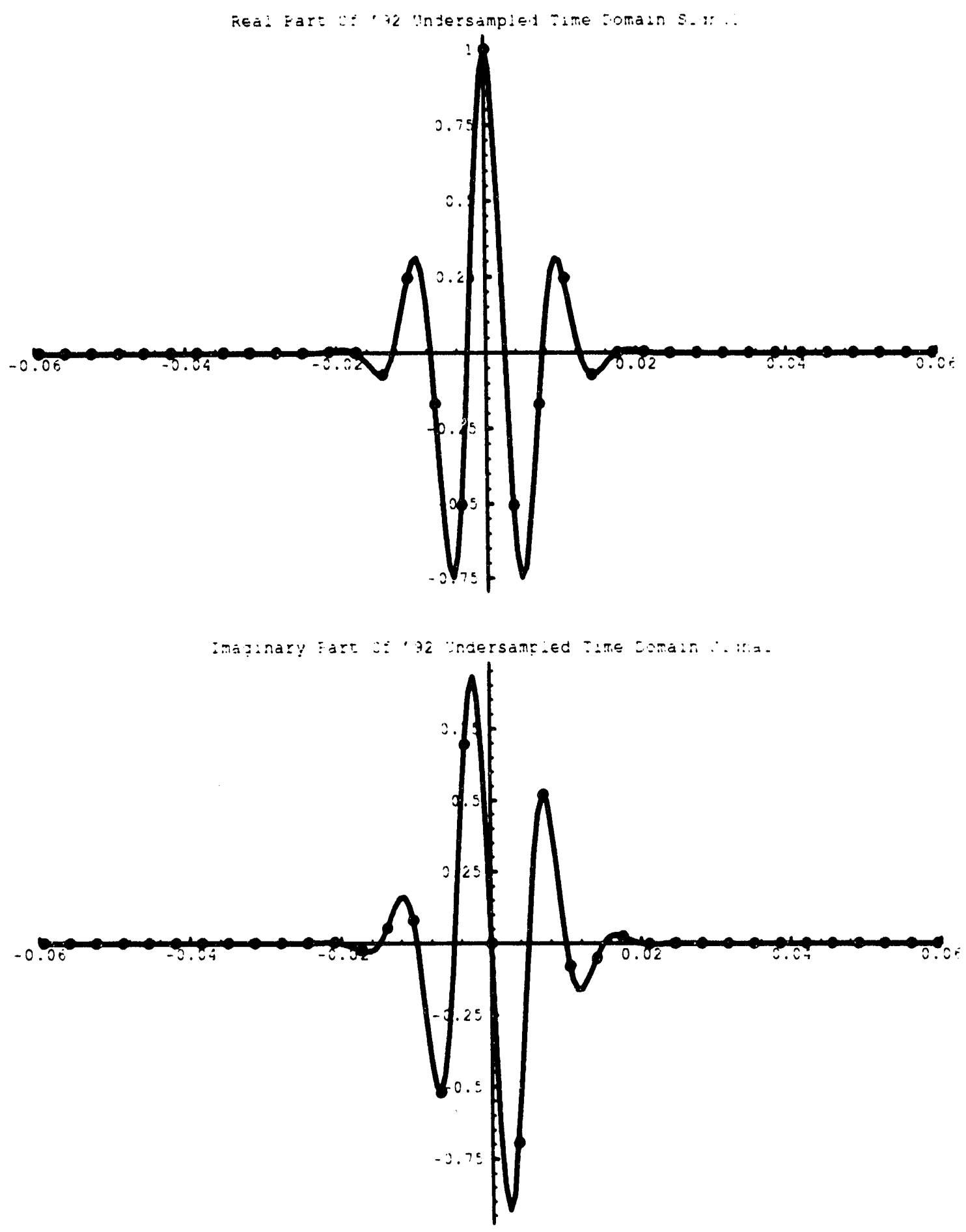

Figure 10: Real and imaginary parts of the analytic time domain signal, $S(t)$. and the discrete signal sampled at the 1992 interval of $3.5 \mathrm{~ms}$. Note: only the center part of the signal is shown. there are actually 128 poilts which have been truncated from the graph because they are zero. 


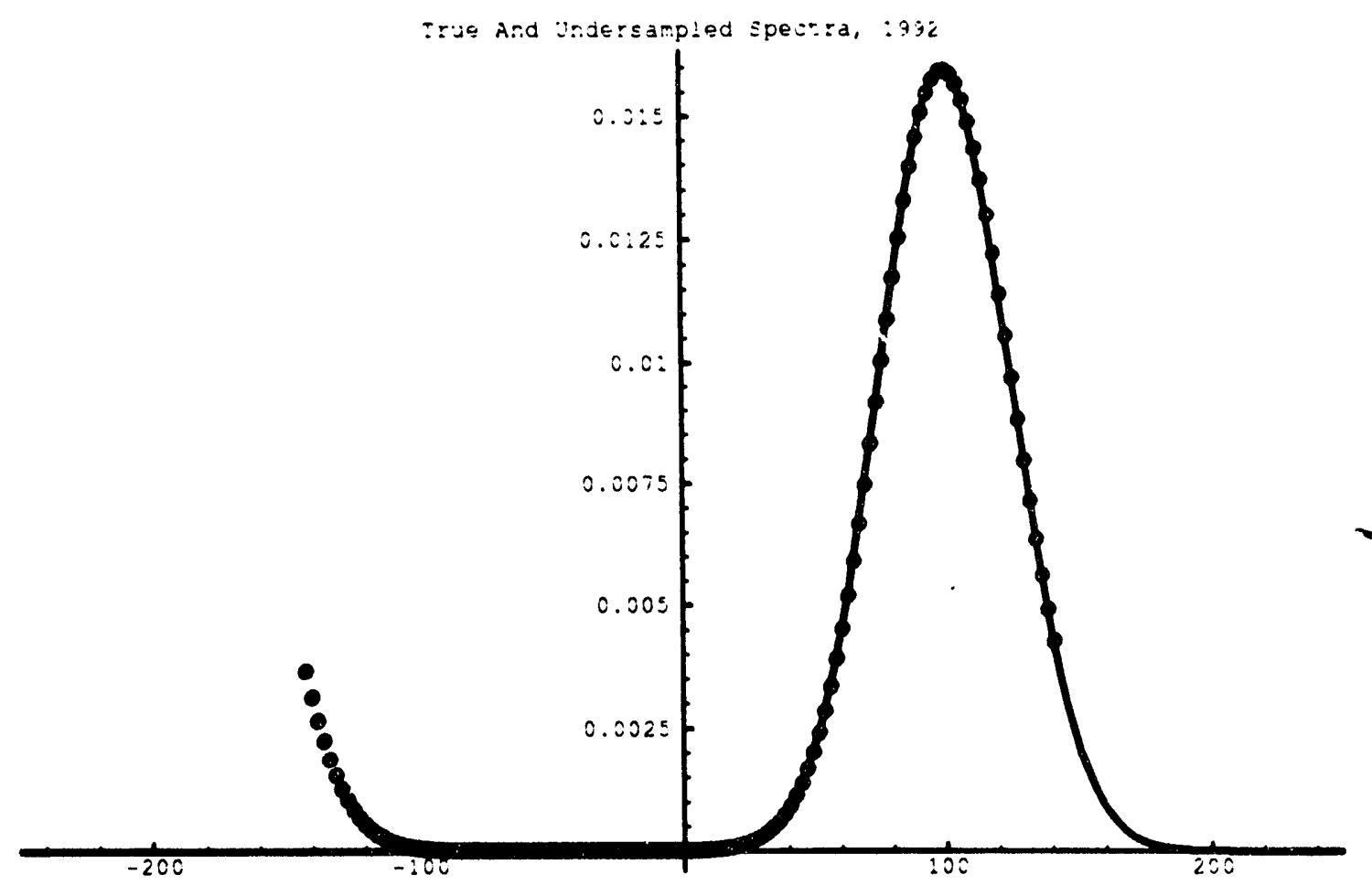

Figure 11: The manufactured Doppler spectrum and 1992 aliased disrete counterpart.

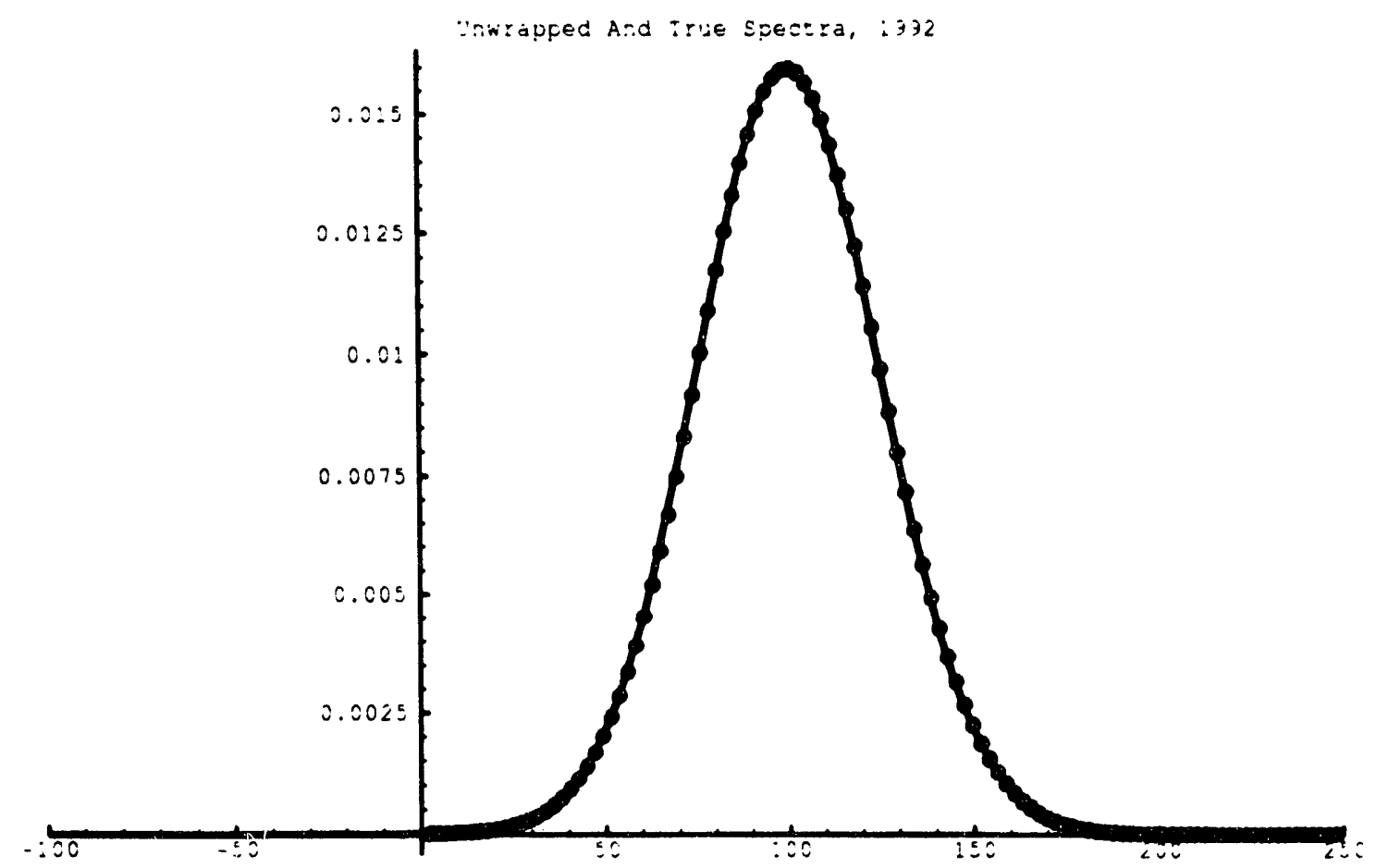

Figure 12: The manufactured Doppler spectrum and 1992 unwrapped sampled spectrum. The cut was nade. at $0 \mathrm{~Hz}$. 

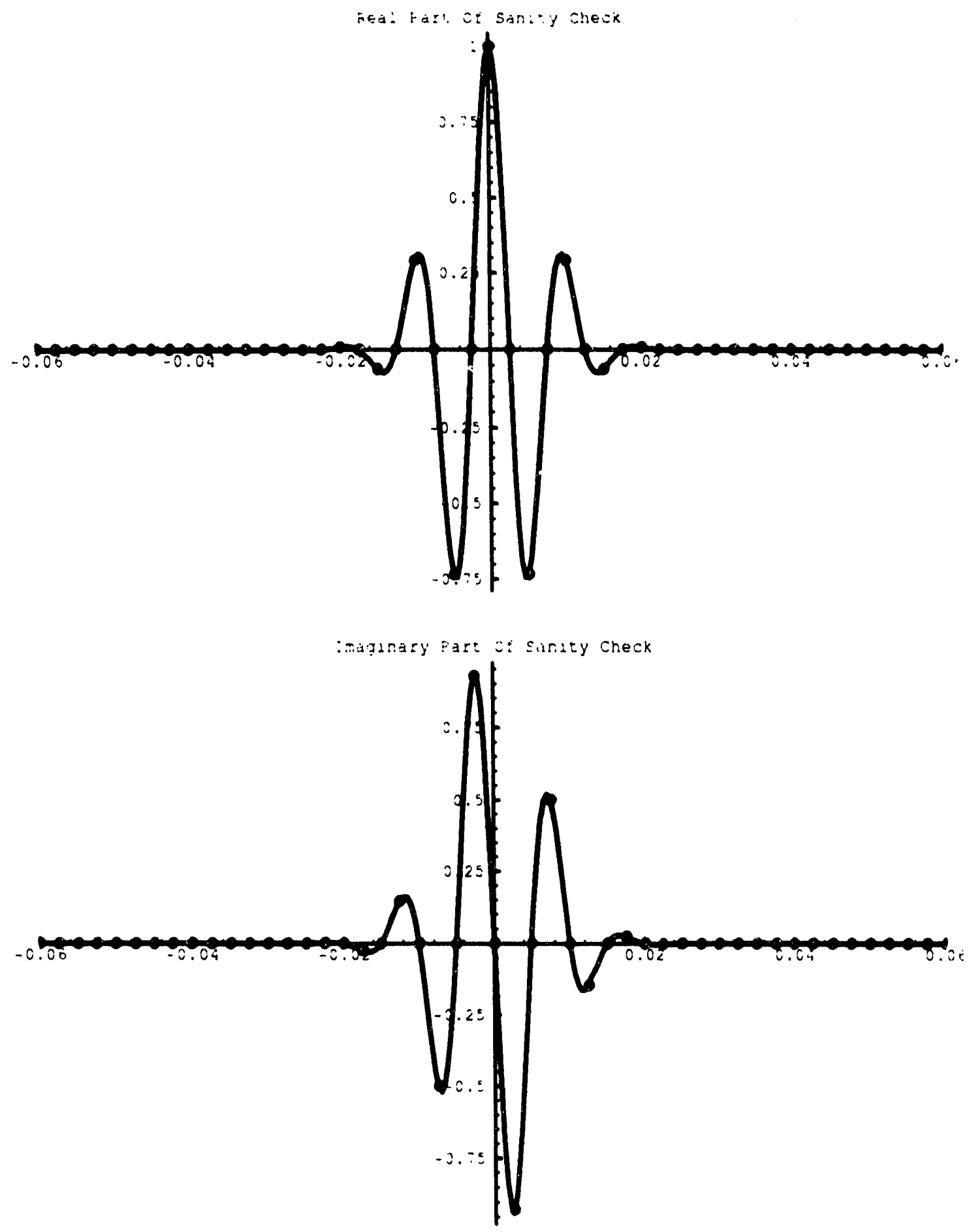

Figure 13: Real and imaginary parts of the analytic time domain signal, $S(t)$. and the sanity check sampled at $400 \mathrm{~Hz}$. The signal is well sampled. Note: only the center part of the signal is shown, there are actuall! 128 points which have been truncated from the graph because they are zero 


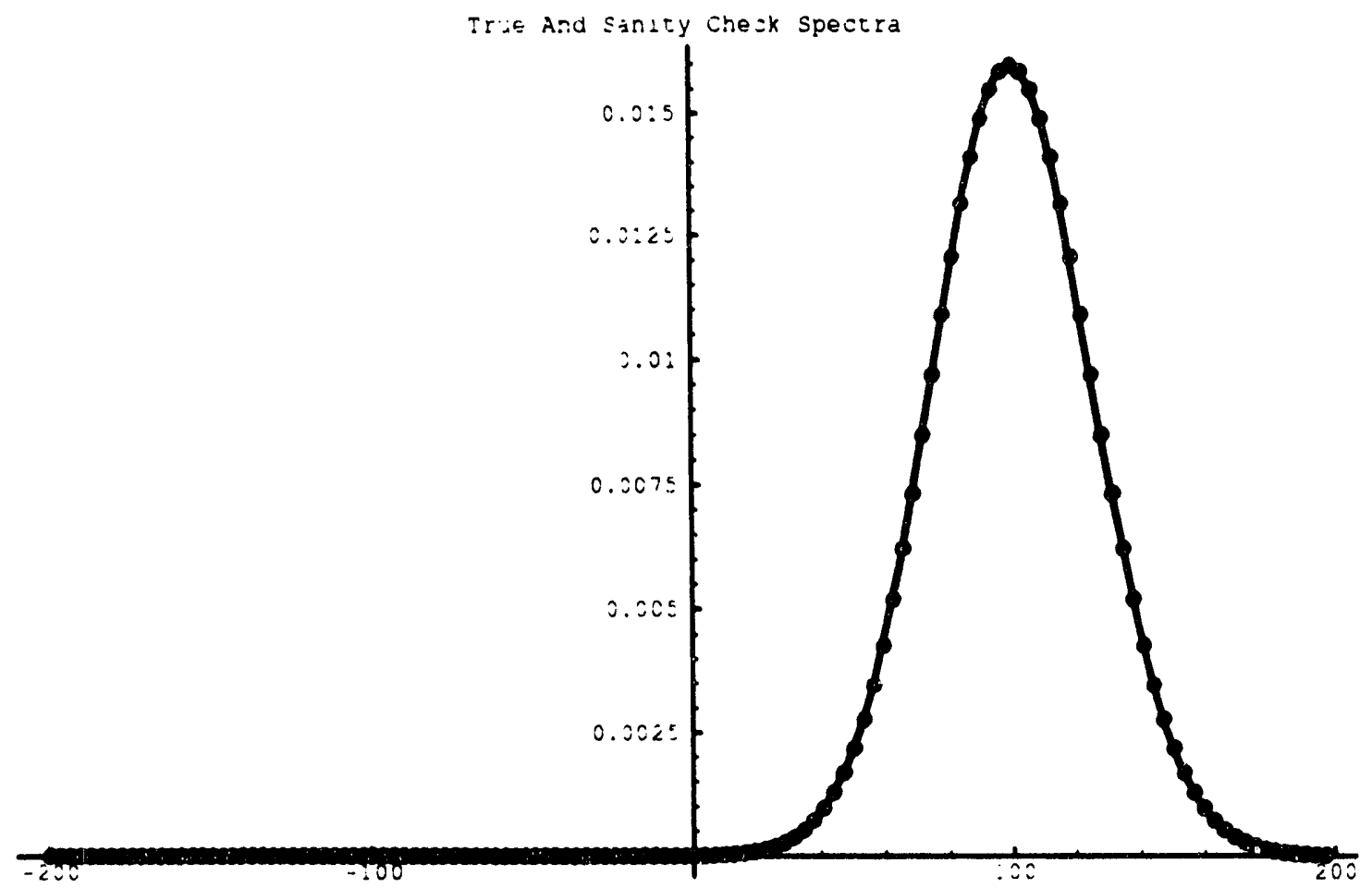

Figure 14: The manufactured Doppler spectrum and 1992 aliased discrete counterpart. 

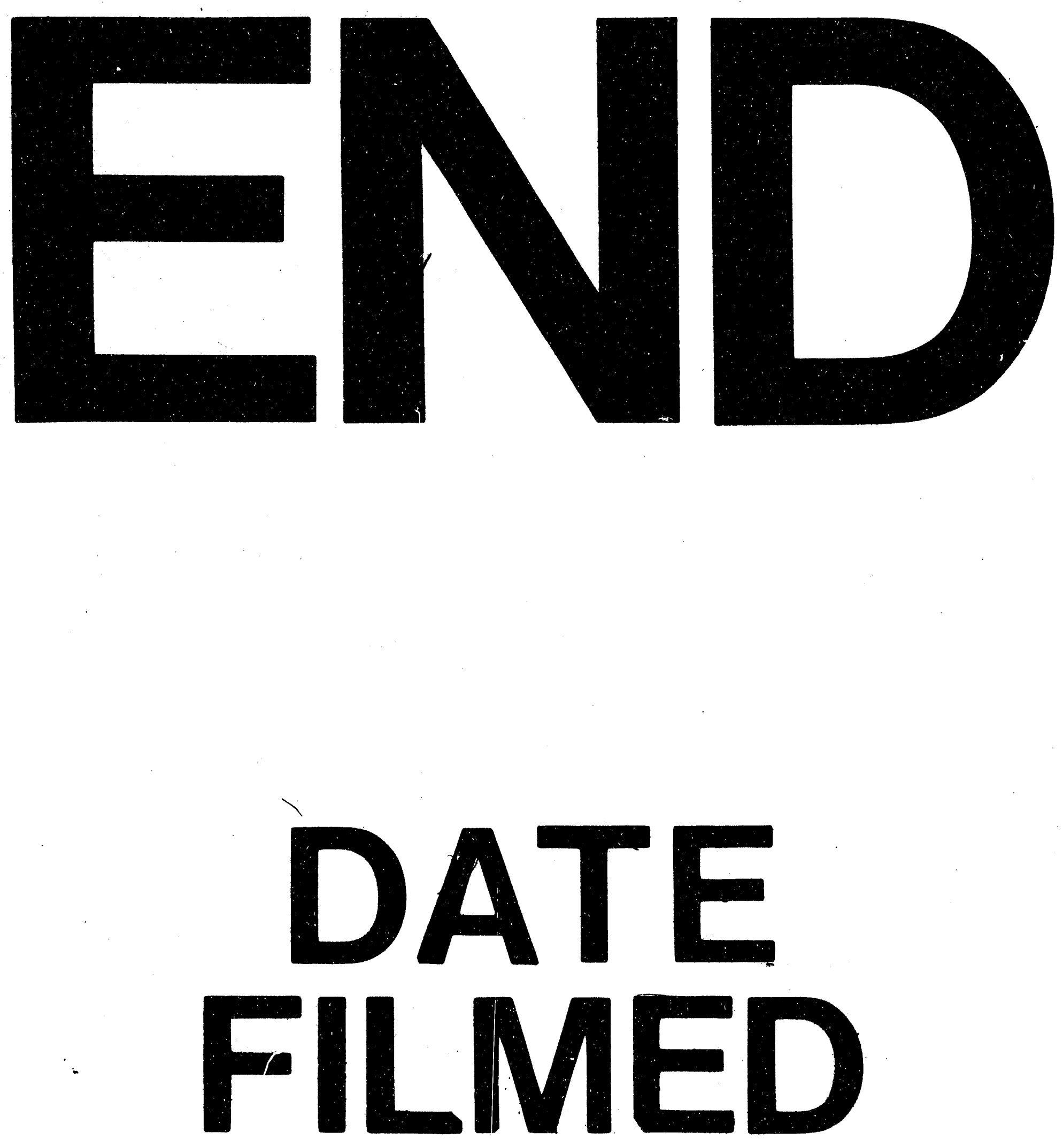

1

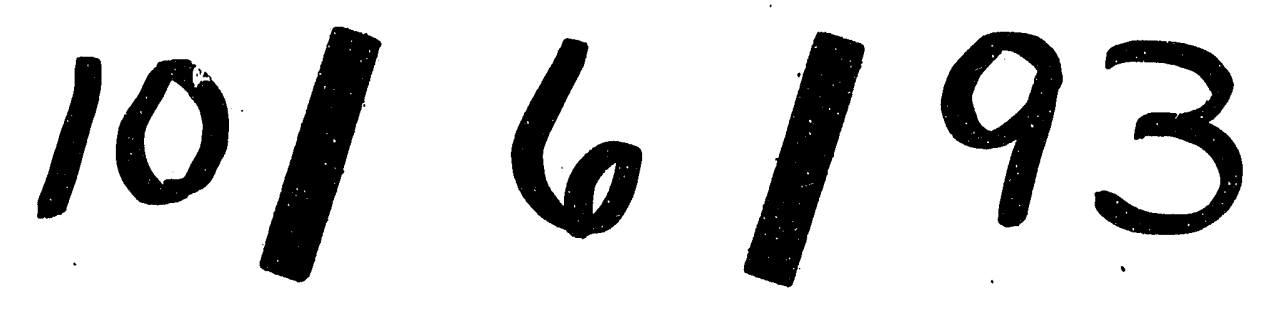


